\title{
Real-Time Detection and Measurement of Cracks in Fatigue Test Applications
}

\author{
Thomas Thurner \\ Institute of Lightweight Design, Graz University of Technology, Graz, Austria, \\ thomas.thurner@tugraz.at
}

\begin{abstract}
:
The detection of crack initiation and the observation of crack growth are of great importance for evaluating the fracture mechanics and fatigue behavior of mechanical components and structures. The proposed method targets on detection and measurement of cracks on metallic surfaces where initial artificial cracks have been applied to the specimen's surface prior to dynamic loading in a test rig. Applying a pre-test surface treatment in terms of polishing the investigation area on the specimen enables for highest resolution and accuracy for crack detection and crack length measurement with a resolution in the order better than $0.05 \mathrm{~mm}$ over a crack length of up to total $10 \mathrm{~mm}$ and more. The developed measurement system operates in real time during cyclic loading of the test unit within the test application. Load-triggered illumination by high-power LEDs together with load-triggered image acquisition by a high resolution digital camera enables the real-time observation of crack development on the investigated surface area. The implementation of the system on a real time hardware enables the robust measurement of crack lengths on-site and in real-time. The developed system achieves impressive real-time measurement performance for crack detection and crack length estimation, in combination with the advantage of non-contacting operation and highly desired on-site measurement capabilities during dynamic load experiments.
\end{abstract}

Key words: Crack detection, crack measurement, real time, optical measurement.

\section{Introduction}

The experimental validation of fatigue properties of mechanical components and structures are of greatest importance the development and realization of most mechanical components and structures, especially for mechanical systems in safetyrelated applications such as the development of railway vehicles. The initiation of cracks in mechanical components and structures can be considered as a critical state for the fatigue lifetime of the overall mechanical system, since after initiation of the first crack the lifetime of the certain component will in most cases be dramatically reduced.

Research in fracture mechanics [1, Part A, Section 5] focuses on the understanding and the mathematical description of crack initiation and the further crack growth during defined load situations. This research is based on analytical, simulation and experimental techniques. Practical experiments such as dynamic tests provide a mean for direct evaluation of fatigue properties and fracture phenomena, and are essential for the development of mathematical descriptions and simulation models. The on-line characterization of cracks within load experiments are thus important for conducting such tests with satisfying results.

The experimental observation of crack initiation and crack growth demands for accurate measurement techniques for the robust detection of cracks and the ongoing sensing of the crack length evolution. Since such experiments are always dynamic load tests on specialized test equipment, the possibility of an on-site measurement of crack phenomena directly on the investigated test specimen inside the testing machine is important.

This paper describes a new technique for the precise and high resolution measurement of crack initiation and evolution on metal surfaces in real time. The method is based on the optical observation of the specimen's surface under special illumination conditions, where prior to the test an initial notch as a well-defined starter point for cracks has been positioned on the polished metallic surface of the specimen. The developed technique provides the accurate measurement of crack length with a resolution in the order of $0.01 \mathrm{~mm}$ and better over crack lengths of $10 \mathrm{~mm}$ and more. 
In contrast to existing methods the high measurement resolution of the developed system can be achieved in-situ in a noncontacting manner, with the possibility for real time crack detection and crack length measurement during continuous cyclic loading of the specimen in dynamic testing machines.

\section{State of the Art: Measurement of Crack Initiation and Crack Growth}

Several techniques for the visualization and measurement of crack initiation and crack growth are existing. Direct visualization of cracks can be achieved by liquid penetrant testing and magnetic particle testing [2]. Ultrasonic and eddy current techniques allow for quantifiable results for crack length and crack depth [2],[3]. Optical whole field techniques such as e.g. speckle-interferometry (ESPI) or digital image correlation (DIC) allow for access to $2 \mathrm{D}$ or $3 \mathrm{D}$ deformation fields on the specimen [4],[1, Part C]. Extensometers and clip gauges are used to directly access surface deformation by the displacement of two measurement points $[1$, Part B]. Strain gauges are used to access local stress distributions and changes in the appearing material stress [1, Part B]. Further strain gauge types have been developed (crack propagation gauges) to directly measure crack growth as segmented elements, which are distributed by commercial companies such as e.g. HBM (Hottinger Baldwin Messtechnik, Germany) and Vishay (Vishay Precision Group, Inc, USA). Further techniques for crack related measurements are photoelasticity [1, Section 5.6], radiographic testing [2], the method of caustic [1, Section 5.6], acoustic emission [5], and thermal testing / thermography [6].

\section{Measurement Principle}

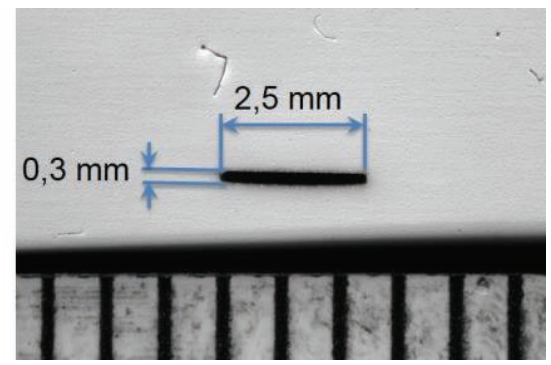

Fig. 1. Initial notch of size $2.5 \times 0.3 \mathrm{~mm}^{2}$ as provided on certain test specimen for the investigation crack initiation and crack growth in steel. The specimen surface has been covered by white painting.

The target application of the developed method is the in-situ detection and observation of cracks during certain fatigue tests, where prior to the tests small initial cracks of defined geometry have been introduced on the specimen's surface. Such experiments are primarily important for research of mechanical fracture mechanics aiming in understanding the evolution of cracks under given dynamic load situations and the development of simulation models for calculating crack initiation and crack propagation in real world components.

Figure 1 shows the geometry of a tiny artificial notch which has been manufactured into the relevant surface area of the test specimen. The notch of size $0.3 \times 2.5 \mathrm{~mm}^{2}$ has been eroded into the specimen's surface prior to the experiment. Underneath the notch a part of the millimeter raster of a conventional tape measurement is visible. The artificial notch acts as well defined starter point for evolving cracks to provoke and investigate the process of crack initiation and crack growth for various load scenarios.

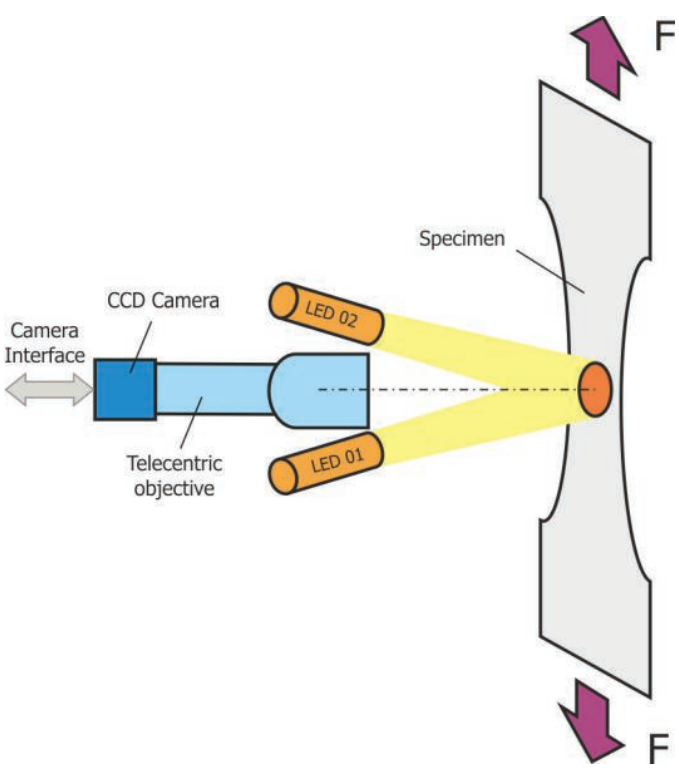

Fig. 2. Optical Setup of the crack measurement system: The interesting surface area on the loaded test specimen is illuminated by two LED spots of high light intensity. A digital camera with attached telecentric objective acquires images of the pretreated surface area under LED illumination.

The overall optical setup of the proposed measurement system is shown in Figure 2. A high resolution digital camera images the relevant area on the mechanically loaded test specimen by a telecentric objective. Due to the high telecentricity and the high accuracy of the used objective, a high geometric imaging performance with negligible image distortions at a constant magnification factor is achieved. Thus relative distance measurements within the imaging area can be made at high accuracy with very simple calibration efforts - such as the imaging of a scale standard and estimation of the real magnification factor. 
The test specimen is loaded with cyclic tensile load $(R=0.1)$ on a conventional fatigue testing machine. Other load types such as bending loads, cyclic tensile/compression load or torsional loads can are also possible, depending on the needed load type and the available testing machine.

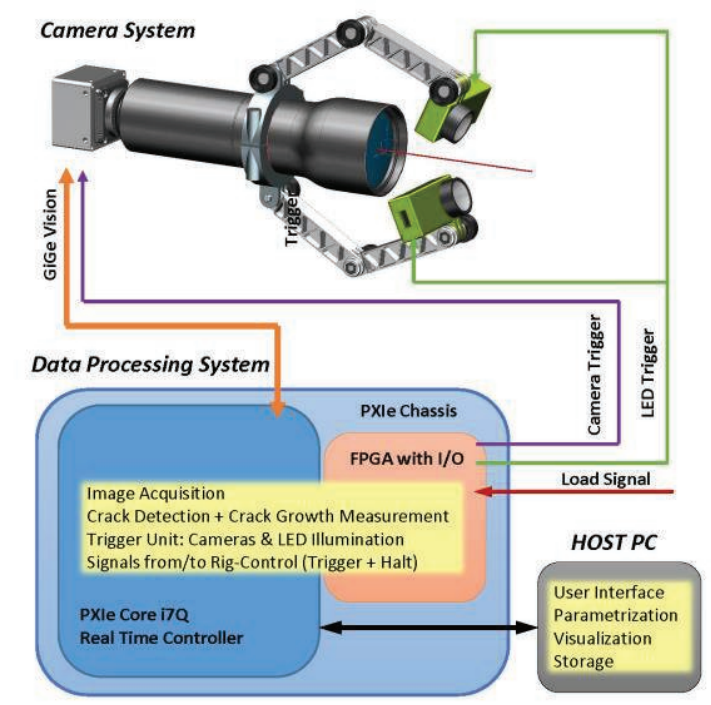

Fig. 3. System diagram of the developed measurement system. A real time system acquires and analyzes images from the loaded specimen, where a trigger unit ensures load-synchronized triggered stroboscopic illumination and image acquisition.

An important aspect of the developed system is the possibility of the in-situ real time measurement possibility for crack phenomena. This is achieved by introducing load triggered surface illumination and image acquisition, based on deterministic trigger events deduced from analog load signals from the testing machine itself. Figure 3 shows the overall system diagram with the digital camera with attached objective and two LED strobe projectors, interfaced by the real time processing system. This real-time system acquires and processes images from the digital camera, and a FPGA system is used for obtaining loading-synchronized deterministic trigger events for pixel integration on the camera and synchronized stroboscopic LED illumination. Any analog input signal - from the loading machine or from strain gauges directly applied to the specimen - can be used for creating valid trigger events. Using loadtriggered image integration and acquisition allows for stationary observation of a single load state within fast dynamic test applications.

The image acquisition and data processing on a real time system allows for highest reliability and determinism of the overall system, such that the measurement system can autonomously operate over long test periods, always providing means for interaction with the test machine controller by analog or digital signals. An important scenario is the online output the estimated crack length as an analog voltage signal for easy integration of crack information in existing measurement/control systems, or the detection of crack initiation to halt the test machine or to online change test loads based on the actual crack length.

The connected Host PC in the diagram in Figure 3 is needed for parametrization of the real time system, data visualization, and image data storage for long-term measurements.

\section{Surface Treatment and Surface Imaging Conditions}

Figure 4, left image, shows a photograph of a test specimen with included initial notch (inside orange circle). Pre-test specimen treatment by polishing its surface to a surface roughness of $\mathrm{Ra}=0.1 \mu \mathrm{m}$ and better is used to robustly visualize even smallest cracks evolving from the initial notch structure.

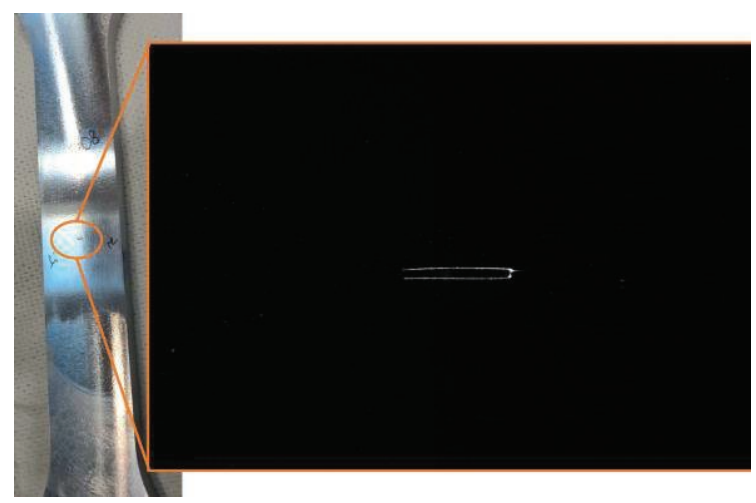

Fig. 4. Test specimen (left) for experimental investigation of crack evolution on different steel grades. The right image shows a magnified picture of the polished and illuminated surface around the initial notch, as used throughout the experiments.

The large right-side image in Figure 4 shows the image of the LED-illuminated polished surface of the test specimen, where only the edges of the notch are visible inside the illuminated surface area. The polished surface reflects all incoming light without any light scattered back into the camera (compare Figure 3). If there is a crack, the illuminating light is being diffracted and scattered towards the camera objective by the small crack dimensions, so that the camera pixels can sense light information from the associated crack positions on the specimen. 


\section{Realized Measurement System}

The measurement system introduced in the previous sections has been realized for characterization of the crack evolution on steel specimen on a dynamic tensile testing machine. The load frequency for this application is given by $30 \mathrm{~Hz}$, making it impossible for non-triggered image acquisition to acquire sharp images. Since the crack only slowly grows over thousands of load cycles during such dynamic loading tests, it is sufficient to utilize only a single trigger event per second for image acquisition and data processing. Thus we obtain crack data with a deterministic update rate of $1 \mathrm{~Hz}$, which is by far sufficient for the target applications of the developed method.



Fig. 5. Realized crack measurement system attached on a conventional dynamic tensile loading machine, consisting of a high resolution CCD-camera with attached telecentric objective, and two illuminating $L E D$ flashlights.

A 12MP CCD camera of MatrixVision (mvBlue COUGAR-XD 1212a) with a resolution of $4250 \times 2838$ pixel of size $3.1 \times 3.1 \mu \mathrm{m}^{2}$ has been used to provide a high measurement resolution over a wide imaging range. A telecentric objective of optical magnification $\mathrm{M}=0.9$ has been attached to the camera. The field of view of the camera system is then given by a width of $15 \mathrm{~mm}$. Two LED flashlights (in-house development) are attached above and below of the objective, to illuminate the test specimen at the pre-treated polished surface area with initial notch. The real time measurement application for image acquisition and data processing has been implemented in LabVIEW RT on a LabVIEW PXle Core i7 Quad-Core controller NI PXle-8135, integrated inside a PXle system chassis $\mathrm{NI}$ PXIe-1071. The digital camera is interfaced by GigE Vision connection (Gigabit Ethernet Vision) to the real-time system. A
FPGA board with I/O functionality of type NI PXI-7841R is included inside the PXle system for trigger functionality and analog/digital signal I/O of the real time system.

The implemented application allows for triggered image acquisition with an image integration time of less than $500 \mu s$. As stated above, only one trigger event per second is being used for image acquisition and data processing - enables direct real-time data processing and visualization on the implemented real-time system.

\section{Results}

The developed measurement method has been verified by real world fatigue tests on special test specimen with an included well-defined artificial starter notch as described in section 3 (Fig. 1) and section 4 (Fig. 4). Following the pre-test polishing treatment of the interesting surface area, the specimen has been clamped inside a fatigue testing machine for conducting cyclic tensile load experiments with load ratio $\mathrm{R}=0.1$ for achieving a mechanical stress amplitude of $100 \mathrm{~N} / \mathrm{mm}^{2}$ in the vicinity of the initial notch.

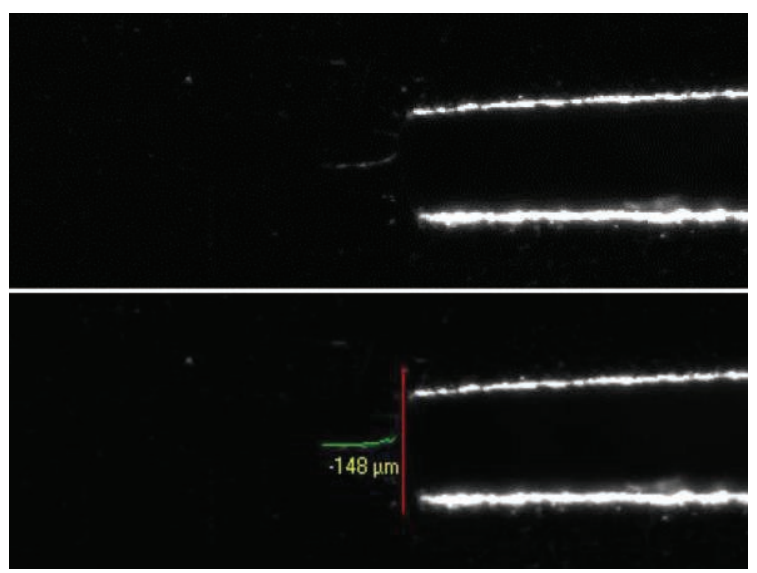

Fig. 6. Upper image: Monochrome image of the illuminated test specimen during dynamic loading. A crack has been appeared starting from the initial notch to the left side, visible as white line within the dark background. Lower image: Result of the on-line crack characterization superimposed on the acquired image: Measured crack length: $148 \mu m$.

The upper image in Figure 6 shows the acquired image for a certain load cycle count, where the bright crack line is already visible within the image. The developed gradient based crack detection algorithm robustly identifies and measures the appearing crack length. After crack length estimation, the recognized crack structure is marked in the visualized image, where the estimated crack length is superimposed as text close to the crack tip. The lower image in Figure 6 shows the estimated crack length and the recognized 
crack line superimposed on the original image. For this acquired image the developed crack measurement system estimates the crack length with $I_{c}=148 \mu \mathrm{m}$. The pixel quantization itself provides an uncertainty of \pm 1 pixel corresponding to a quantization uncertainty of \pm $3.44 \mu \mathrm{m}$ at the specimen surface (for the given combination of camera and objective).

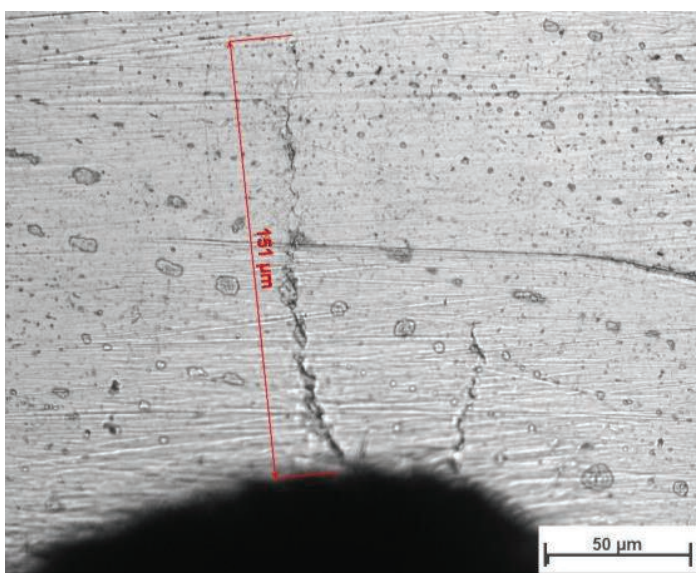

Fig. 7. Investigation of crack structure and measurement of the crack length with a metallographic measurement microscope and an optical magnification factor of $M=500$. Measured crack length: $151 \mu \mathrm{m}$. (Institute of Material Science and Welding, Graz University of Technology).

The performance of the developed crack measurement has been compared to measurement results obtained by a commercial high precision metallurgic microscope. Of course the specimen has to be released from the loading machine and transferred to the measurement chamber of the metallurgic laboratory for this investigation. Figure 7 shows the results from the metallurgic microscope at a magnification factor of $M=500$. The crack is clearly visible within the microscope image (together with a closely located second small crack), and the crack length has been measured as $I_{c}{ }^{\prime}=151 \mu \mathrm{m}$ by the calibrated microscope system. The whole crack has thus been resolved and recognized by the developed measurement system, demonstrating the high measurement capabilities of the developed methodology.

Throughout separate dynamic load tests with different test specimen and different crack lengths from $100 \mu \mathrm{m}$ up to $4 \mathrm{~mm}$, such evaluations off-site with an external optical measurement microscope has been accomplished, where observed crack length has always been within the range of maximum \pm 1 pixel of the camera discretization. The obtained results demonstrate the high measurement performance of the novel experimental technique for real-time characterization of crack growth in cyclic loading situations.

\section{Conclusion}

The proposed measurement method provides a robust and accurate detection of crack initiation and the highly resolving measurement of crack lengths on metallic specimen with a pre-treated surface in dynamic loading scenarios. In contrast to existing methods the developed methodology provides high measurement resolution by a non-contacting optical setup and real time on-site measurement capabilities: Crack lengths can be robustly and accurate measured during cyclic loading within dynamic load application in a test machine. Alternative measurements of the obtained crack lengths with a commercial measurement microscope validate the high measurement performance of the developed system.

\section{Acknowledgements}

This work has been supported by the international joint research project "Eisenbahnfahrwerke III", funded by the Austrian COMET funding program (K2 projects $\mathrm{MCL}$ and $\mathrm{ViF}$ ), with the industrial and academic partners Alstom Transport Deutschland, Bochumer Verein Verkehrstechnik, Gutehoffnungshütte Radsatz, Siemens AG Österreich, Stadler Pankow, Voith Turbo, Erich Schmid Institute for Materials Science (Freiburg), Fraunhofer Institute for Mechanics of Materials (Austrian Academy of Sciences), Materials Center Leoben, Virtual Vehicle Research Center Graz, Institute of Plant Engineering and Fatigue Analysis (TU Clausthal), Institute of Mechanical Engineering (University of Leoben) and the Institute for Lightweight Design (TU Graz).

\section{References}

[1] W. N. Sharpe, jr, (Ed.), Springer Handbook of Experimental Solid Mechanics, Springer Science, LLC New York, 2008, ISBN 978-0387268835.

[2] L. Cartz, Nondestructive Testing : Radiography, Ultrasonics, Liquid Penetrant, Magnetic Particle, Eddy Current, ASM Intl; ISBN: 0871705176.

[3] J. Krautkramer, and H. Krautkramer, Ultrasonic Testing of Materials, 4th/revised edition, Springer Verlag, November 1990, ISBN: 0387512314.

[4] P. Rastogi, and D. Inaudi, Trends in optical Nondestructive testing and inspection, Elsevier, Amsterdam, 2000, ISBN 978-0080430201.

[5] R. K. Miller (Ed.), E. v. K. Hill (Ed.), P. O. Moore (Ed.), Nondestructive Testing Handbook, Third Edition: Vol. 6, Acoustic Emission Testing, Amer. Soc. for NDT, 2005, ISBN-13: 978-1571171061

[6] X. Maldague (Ed.), P. O. Moore (Ed.), Nondestructive Handbook, Infrared and Thermal Testing, Volume 3, 3rd edition, Columbus, Ohio, ASNT Press, 2001. 